\title{
Fresh Frozen Plasma in Hereditary Angioedema Crisis: to Give or Not to Give?
}

\author{
Jamal Belkhouribchia ${ }^{1}$, Sara Nguyen $^{2}$ \\ ${ }^{1}$ Department of Internal Medicine, UZ Brussel, Brussels, Belgium. \\ ${ }^{2}$ Department of Dermatology, UZ Brussel, Brussels, Belgium.
}

Received: $21 / 07 / 2015$

Accepted: $11 / 08 / 2015$

Published: 10/09/2015

How to cite this article: Belkhouribchia J, Nguyen S. Fresh frozen plasma in hereditary angioedema crisis: to give or not to give?. EJCRIM

2015;2:doi:10.12890/2015_000253

Conflicts of Interests: The authors declare that there are no competing interests.

Patient's Consent: The authors declare they obtained patient's consent for publication.

This article is licensed under a Commons Attribution Non-Commercial 4.0 License

\section{ABSTRACT}

Objectives: Fresh frozen plasma (FFP) has been used in angioedema crises, however there is a risk of aggravating the symptoms as well as transmitting infections. In this report, the authors emphasize the dangers of this therapy.

Materials and methods: A 25-year-old woman with hereditary angioedema (HAE) was treated with FFP after which her symptoms escalated. Results: Administration of purified C1-inhibitor (C1-INH) resulted in relief of her symptoms.

Conclusions: FFP is to be avoided in a HAE crisis. Newer therapies for angioedema are preferred.

\section{LEARNING POINTS}

- Angioedema should be considered when sudden onset facial edema is not associated with urticaria or pruritus, and does not improve with glucocorticoids and $\mathrm{H} 1$-antihistamines.

- Fresh frozen plasma (FFP) is to be avoided in HAE crises as it can aggravate symptoms and carries the danger of transmitting infectious diseases; it can also induce allergic transfusion reactions, transfusion-related acute lung injury and circulatory overload.

- Recombinant or human plasma-derived C1-inhibitor (C1-INH), icatibant or ecallantide are the first line treatments in hereditary angioedema attacks.

\section{KEYWORDS}

Hereditary angioedema, angioedema, fresh frozen plasma, facial swelling.

\section{INTRODUCTION}

Hereditary angioedema (HAE) is a rare autosomal dominant disease that is characterized by temporary swelling of the skin or mucosal tissue. The underlying etiology is a deficiency or dysfunction of $\mathrm{C} 1$-inhibitor (C1-INH) ${ }^{[1]}$. HAE is often mistakenly treated as an Immunoglobulin $\mathrm{E}$ (IgE)-mediated allergic reaction. Fresh frozen plasma (FFP) has been used successfully in the past for the treatment of angioedema when human C1-INH concentrates were not readily available ${ }^{[2]}$. There are, however, significant concerns about the safety of FFP as it can worsen the symptoms and transmit infections. Recombinant or human plasma-derived C1 inhibitor (C1-INH), icatibant and ecallantide are the first line treatment when available).

\section{CASE REPORT}

A 25-year-old woman presented at the emergency department with swelling of the face that started that morning (Fig. 1). She had already 
been seen at an emergency service of another hospital, where she was diagnosed with allergy and treated with $\mathrm{H} 1$-antihistamines and intravenous glucocorticoids. As the swelling worsened, she came to our emergency service for a second consultation. She had two similar episodes of swelling the past year that spontaneously subsided. There was no family history of swelling nor urticaria, and she had no known allergies. She did not take any medications nor did she take oral contraceptives. Her medical history was unremarkable. Blood pressure was $136 / 86 \mathrm{mmHg}$; pulse was $96 /$ minute; and temperature was $36.0^{\circ} \mathrm{C}$. Her face and eyelids were swollen; there was no urticaria; and she did not have problems breathing at presentation. Since the earlier treatment of H1-antihistamines and glucocorticoids did not improve her symptoms, the diagnosis of an allergy was discarded and the possibility of angioedema was considered. As other treatment options were not readily available, she received two units of FFP after which her symptoms aggravated within 30 minutes (Fig. 2). She also developed difficulty speaking. Subsequently, Human plasma-derived C1-INH was given and resulted in a remarkable improvement of her symptoms, with the swelling subsiding within one hour (Fig. 3). Blood analyses (drawn before the FFP was given) showed a low complement 4 (of 4.9 mg/dL (1128); a low C1-INH concentration of $5 \mathrm{mg} / \mathrm{dL}$ (20-34); a low C1-INH function of 22\% (70-130); and a normal C1q level of 0.22 g/L (0.10-0.25): the diagnosis of a type 1 hereditary angioedema was thereby confirmed.

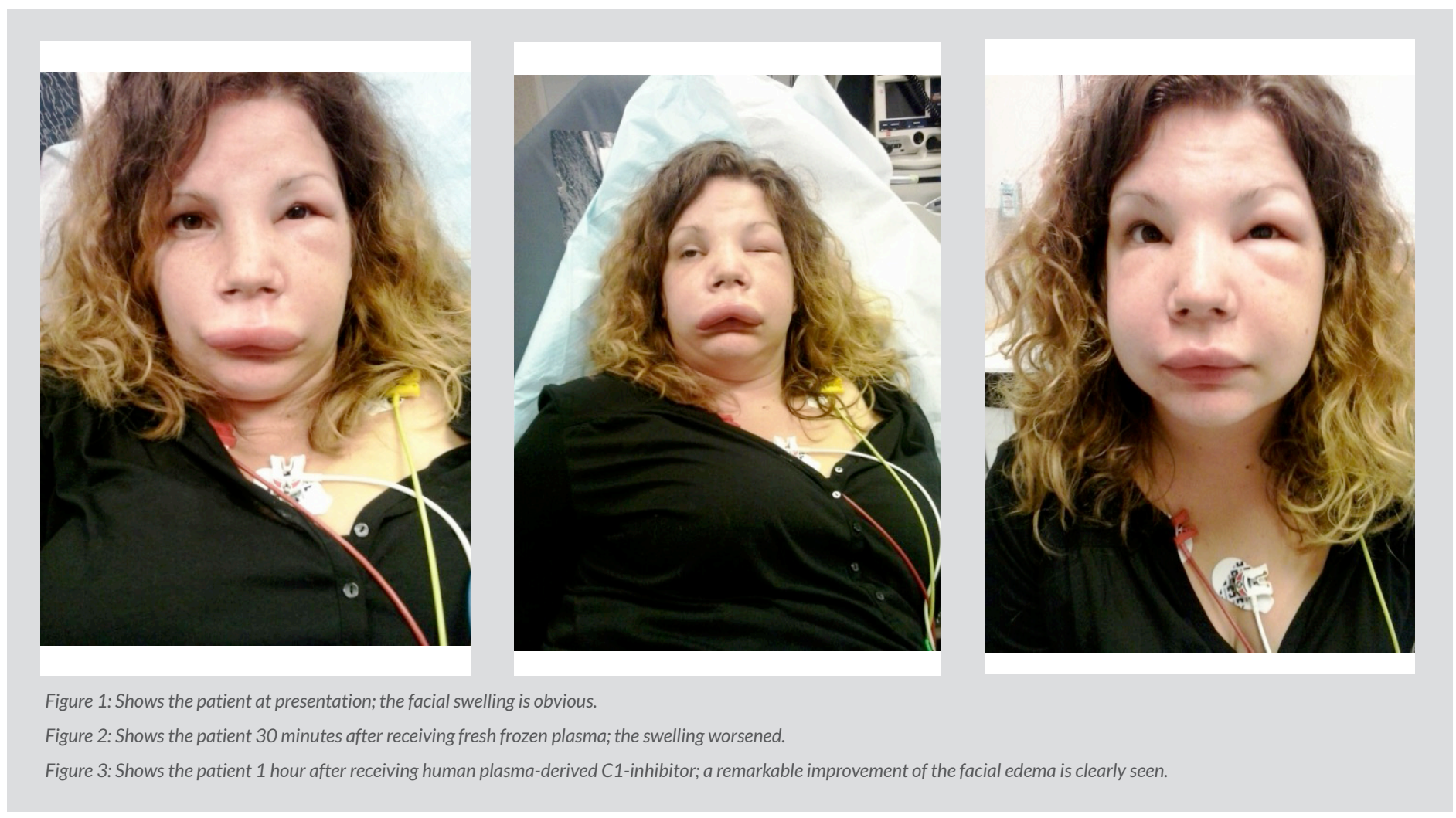

\section{DISCUSSION}

HAE is an autosomal dominant disease that was first described by Sir William Osler in 1888 in the American Journal of the Medical Sciences. The underlying mechanism is a deficiency of C1-INH. This disease manifests itself by recurrent episodes of subcutaneous or submucosal edema, typically without itching or urticaria. Swelling can involve any part of the body. The symptoms tend to worsen over the first 24 hours, after which they slowly subside over 48 to 72 hours. Laryngeal edema is a feared entity as it can lead to asphyxiation if not treated timely and adequately. Two types of HAE are distinguished. Type $1 \mathrm{HAE}$ is characterized by low levels of antigenic C1-INH as well as a low functional activity, and constitutes $85 \%$ of cases. Type 2 HAE is seen in 15\% of HAE patients; a normal antigenic C1-INH is seen with low functional levels. Both types are clinically indistinguishable. One should be mindful of the diagnosis of HAE in patients with recurrent episodes of cutaneous or mucosal edema without itching or urticaria. A family history of angioedema is often the case, though up to $25 \%$ of patients have a de novo mutation of the $\mathrm{C} 1-\mathrm{INH}$ gene, as was the case in our patient. Diagnostic confirmation is obtained by laboratory tests. HAE patients have a low antigenic $\mathrm{C} 4$ level, therefore $\mathrm{C} 4$ is a useful screening test. First line treatment of HAE attacks involves replacement therapy with intravenous Human plasma-derived C1-INH, recombinant human C1-INH, subcutaneous icatibant (a bradykinin B2-receptor antagonist) or subcutaneous ecallantide (a kallikrein inhibitor) ${ }^{[3]}$. The use of subcutaneous purified C1-INH is under investigation at the moment. FFP is 


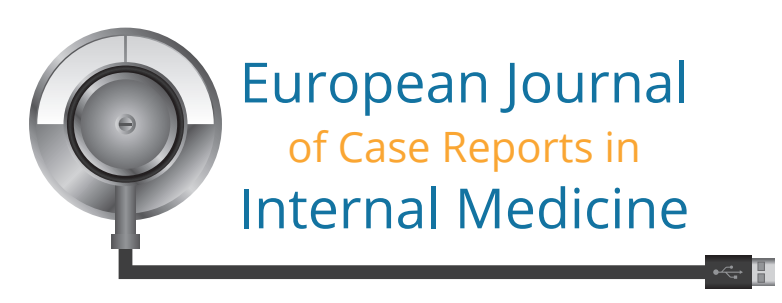

to be avoided as is contains contact proteins that can aggravate angioedema symptoms; there is also a risk of transmitting infections with FFP[4]. Other risks involved in the use of FFP include: transfusion-related acute lung injury; transfusion-associated circulatory over-load; allergic and/or anaphylactic reactions; and to a lesser extent febrile nonhemolytic transfusion reactions; red blood cell alloimmunization; and hemolytic transfusion reactions ${ }^{[5]}$. Although the newer therapies mentioned above are expensive, their benefit outweighs the risk that is taken with the administration of FFP. Therefore, today FFP as treatment for HAE attacks should only be considered in severe cases of angioedema when first line treatment options are not available.

\section{CONCLUSION}

In acute HAE exacerbations, C1-INH replacement therapy is the mainstay of management; icatibant and ecallantide are also considered effective and safe. FFP is to be avoided as symptoms could worsen due to the risks of allergic transfusion reaction and transmitting infection. We do not recommend its use in angioedema as better and safer options are available.

\section{REFERENCES}

1. Zuraw BL. Hereditary angioedema. NEJM 2008 Sep 4;359:1027-36.

2. Tang R, Chen S, Zhang HY. Fresh frozen plasma for the treatment of hereditary angioedema acute attacks. Chin Med Sci J 2012;27:92-5

3. Zanichelli A, Mansi M, Periti G, Cicardi M. Therapeutic management of hereditary angioedema due to C1 inhibitor deficiency. Expert Rev Clin Immunol 2013;9:477-88.

4. Kalaria S, Craig T. Assessment of hereditary angioedema treatment risks. Allergy Asthma Proc 2013;34:519-22.

5. Pandey S, Vyas GN. Adverse effects of plasma transfusion. Transfusion 2012;52 Suppl 1:65S-79S. 